\title{
Calibration of the SCIAMACHY instrument on the ESA-ENVISAT satellite
}

\author{
Marcel R. Dobber \\ TNO Institute of Applied Physics (TNO-TPD), P.O. Box 155, 2600 AD Delft,
}

The Netherlands.

\begin{abstract}
This document describes the calibration and performance of the SCIAMACHY instrument, to be launched on ESAENVISAT in 2000, after the main on-ground calibration and performance verification phases. A number of calibration and performance parameters will be discussed and results will be shown.
\end{abstract}

Keywords: Calibration, Polarisation, Spectrometer, Hyperspectral, Radiometric calibration.

\section{INTRODUCTION AND INSTRUMENT DESCRIPTION}

The SCIAMACHY (SCanning Imaging Absorption spectroMeter for Atmospheric CHartographY) instrument, to be launched on the ESA-ENVISAT earth environmental satellite in November 2000, has been designed to measure atmospheric trace gases and aerosols in order to monitor climate processes and climate changes. It covers the ultraviolet to infrared wavelength range from $240-2385 \mathrm{~nm}$ with a high spectral resolution.

The optical layout of the spectrometer is shown in figure 1. In brief, the instrument is equipped with two uncoated aluminium scanning mirrors, the azimuth scan mirror (ASM) and the elevation scan mirror (ESM). Via the telescope system and the entrance slit the light is first dispersed by a predisperser prism, which also serves the purpose of directing a percentage of about $13 \%$ of the input light polarised parallel to the entrance slit towards the so-called polarisation measurement device (PMD), a dedicated device to measure the input polarisation of the light and appropriately correct for it (see below). The main optical beam is spatially separated into two ultraviolet channels 1 and 2 through a segmented mirror, two infrared channels 7 and 8 by means of a pick-off prism, whereas the remaining visible-infrared wavelength range continues towards the second level of the instrument, where it is separated into channels 3-6 by means of three dichroic mirrors. Channels 7 and 8 are also separated by a dichroic mirror. Each of the 8 channels is subsequently further dispersed by a grating and imaged by channel optics onto a 1024-element one-dimensional array detector. A number of optical characteristics as calibrated are listed in table 1 . The infrared detectors of channels 6-8 have been designed specifically for the SCIAMACHY project.

\begin{tabular}{||c|c|c|c|c|c||}
\hline Channel \# & $\begin{array}{c}\text { Detector } \\
\text { metarial }\end{array}$ & $\begin{array}{c}\text { Detector } \\
\text { temperature } \\
(\mathbf{K})\end{array}$ & $\begin{array}{c}\text { Wavelength } \\
\text { range }(\mathbf{n m})\end{array}$ & Pixel range & $\begin{array}{c}\text { Spectral } \\
\text { resolution in px } \\
\text { and nm }\end{array}$ \\
\hline 1 & $\mathrm{Si}$ & 206.4 & $240.0-334.3$ & $0-1023$ & 2.0 \\
\hline 2 & $\mathrm{Si}$ & 205.8 & $300.4-412.3$ & $1024-2047$ & 1.9 \\
\hline 3 & $\mathrm{Si}$ & 223.7 & $383.4-628.4$ & $2048-3071$ & 2.0 \\
\hline 4 & $\mathrm{Si}$ & 222.3 & $595.2-812.4$ & $3072-4095$ & 2.0 \\
\hline 5 & $\mathrm{Si}$ & 220.8 & $773.0-1062.6$ & $4096-5119$ & 2.0 \\
\hline 6 & InGaAs & 201.7 & $971.4-1772.4$ & $5120-6143$ & 1.8 \\
\hline 7 & InGaAs & 149.7 & $1934.5-2043.5$ & $6144-7167$ & 2.1 \\
\hline 8 & InGaAs & 146.4 & $2255.3-2385.5$ & $7168-8091$ & 2.1 \\
\hline
\end{tabular}

Table 1: characteristics as built for the 8 main optical channels.

The instrument enables various observational modes both for atmospheric measurements and in-flight calibration purposes. These modes and their characteristics are listed in table 2. The earth radiances can be measured sequentially in both nadir and limb geometry. The observation strategy has been set up to observe the same atmospheric air volume first in limb and subsequently in nadir, with a time delay of about 5 minutes. This enables more accurate retrieval techniques by combining measurement results of both modes. The ENVISAT satellite will fly a sun-synchronous polar orbit with a descending node time of $10.00 \mathrm{am}$. Sun observations are possible over the noth pole in two modes: Firstly solar occultation measurements can be performed, where the sun is observed directly through the atmosphere at different tangential heights. Once the line of sight from the instrument to the sun is above the atmosphere the measurements can be used for instrument calibration 
purposes. Secondly, the sun can be observed via an on-board diffuser. These measurements provide the necessary information for sun-normalised nadir and limb radiance data products, and enable absolute calibration of the irradiance of the sun. The measurement geometry of the sun-over-diffuser illumination is depicted in figure 2 . Near the equator the sun can be viewed directly over the elevation scan mirror. This so-called subsolar calibration mode is used primarily for inflight radiometric calibration in order to monitor degradation of the scan mirrors. Different scanning strategies of the scan mirror(s) can be employed when observing the sun. For the sun-over-diffuser mode the mirrors are not scanning. The azimuth scan mirror is fixed into the correct position, correcting for seasonal effects, while the orbital motion of the satellite causes the illumination conditions to vary in elevation direction. This is corrected for when calibrating the sun irradiance dataproducts. In the other sun-observation modes the elevation scan mirror can scan the instrument instantaneous field of view (IFOV) over the solar disc in order to minimise the impact of solar limb darkening, sunspots and solar flares. The IFOV can scan over the disc within one exposure of the detectors in about 125 milliseconds (fast sweep scanning) or it can scan over the disc in 2 seconds (nominal scanning). During this period the detectors are read out several times to avoid saturation. Finally, staring to the sun centre is also possible. For the sun and moon scanning and for finding them a dedicated sun-follower has been installed on the instrument. In order to sufficiently reduce the light intensity levels for direct solar observations the instrument is equipped with two insertable mechanisms: a neutral density filter (NDF) mechanism, which reduces the light intensity in the visible-infrared channels 3-6 by a factor of about 5 , and a small aperture mechanism which reduces both the light intensity of all channels in a channel-dependent way by about a factor of 3000 and the instantaneous field of view of the instrument. Table 2 lists which measurement modes make use of these mechanisms. Figure 3 shows the as-built transmission characteristics of the NDF, while figure 4 shows the light intensity attenuation per channel of the small aperture mechanism.

\begin{tabular}{|c|c|c|c|c|c|c|c|}
\hline Mode & ESM & $\overline{\text { ASM }}$ & Diffuser & NDF & $\begin{array}{c}\text { Small } \\
\text { aperture }\end{array}$ & $\begin{array}{c}\text { Subsolar } \\
\text { port }\end{array}$ & Mirror scanning \\
\hline Nadir & $\mathrm{X}$ & & & & & & nadir scanning \\
\hline Limb & $\mathrm{X}$ & $\mathrm{X}$ & & & & & limb scanning \\
\hline Solar occultation & $\mathrm{X}$ & $X$ & & $\mathrm{X}$ & $\mathrm{X}$ & & normal \\
\hline Solar calibration & $\mathrm{X}$ & $\mathrm{X}$ & & $\mathrm{X}$ & $\mathrm{X}$ & & $\begin{array}{c}\text { normal, fast } \\
\text { sweep, pointing }\end{array}$ \\
\hline $\begin{array}{c}\text { Sun-over-diffuser } \\
\text { calibration }\end{array}$ & & $\mathrm{X}$ & $\mathrm{X}$ & $\mathrm{X}$ & & & no scanning \\
\hline $\begin{array}{c}\text { Subsolar } \\
\text { calibration }\end{array}$ & $\mathrm{X}$ & & & $\mathrm{X}$ & $\mathrm{X}$ & $\mathrm{X}$ & $\begin{array}{c}\text { normal, fast } \\
\text { sweep, pointing }\end{array}$ \\
\hline Moon occultation & $\mathrm{X}$ & $\mathrm{X}$ & & & & & pointing \\
\hline Moon calibration & $\mathrm{X}$ & $\mathrm{X}$ & & & & & pointing, normal \\
\hline Internal SLS & $\mathrm{X}$ & & & & & & no scanning \\
\hline $\begin{array}{c}\text { Internal WLS } \\
\text { regular }\end{array}$ & $\mathrm{X}$ & & & $\mathrm{X}$ & & & no scanning \\
\hline $\begin{array}{c}\text { Internal WLS } \\
\text { NDF monitoring } 1\end{array}$ & $\mathrm{X}$ & & & & & & no scanning \\
\hline $\begin{array}{c}\text { Internal WLS } \\
\text { NDF monitoring } 2\end{array}$ & $\mathrm{X}$ & & & $\mathrm{X}$ & & & no scanning \\
\hline
\end{tabular}

Table 2: Measurement mode configurations. Symbols and abbreviations are explained in the text.

Moon occultation and calibration measurements are possible over the south polar region in a similar way as for the sun observations. The moon accultation measurements are performed while staring at the centre of the moon, as guided by the sun/moon follower. For moon calibration purposes the IFOV is scanned in 2 seconds over the lunar disc within one exposure of the detectors.

Due to the fact that polarisation sensitive optics have been used in the instrument (most notably the gratings and the dichroic mirrors) the instrument radiometric response is strongly polarisation dependent. The instrument corrects for the polarisation state of the incident light using a dedicated Polarisation Measurement Device (PMD). The first 6 PMD broadband detectors measure the polarisation component parallel to the entrance slit, while the $7^{\text {th }}$ PMD broadband detector measures the polarisation component with a direction of 45 degrees with respect to the entrance slit. The characteristics of the PMD detectors are listed in table 3. 
The instrument radiometric calibration is based on the Stokes vector and Mueller matrix descriptions of polarised light. In short, a Stokes vector describes polarised light as follows:

$$
\left(\begin{array}{c}
\mathrm{I} \\
\mathrm{Q} \\
\mathrm{U} \\
\mathrm{V}
\end{array}\right)=\left(\begin{array}{c}
\text { Intensity Unpolarised Light } \\
\text { Intensity } 0^{\circ}-\text { Intensity } 90^{\circ} \\
\text { Intensity }+45^{\circ}-\text { Intensity }-45^{\circ} \\
\text { Intensity Circular Polarisation }
\end{array}\right.
$$

\begin{tabular}{||c|c|c|c|c||}
\hline \hline PMD \# & $\begin{array}{c}\text { Total wavelength } \\
\text { range (nm) }\end{array}$ & Material & $\begin{array}{c}\text { FWHM wavelength } \\
\text { range (nm) }\end{array}$ & Polarisation direction measured \\
\hline 1 & $280-400$ & $\mathrm{Si}$ & $309-377$ & parallel to slit \\
\hline 2 & $430-570$ & $\mathrm{Si}$ & $450-522$ & parallel to slit \\
\hline 3 & $580-750$ & $\mathrm{Si}$ & $610-701$ & parallel to slit \\
\hline 4 & $740-980$ & $\mathrm{Si}$ & $806-908$ & parallel to slit \\
\hline 5 & $1300-1900$ & $\mathrm{InGaAs}$ & $1505-1642$ & parallel to slit \\
\hline 6 & $2200-2500$ & $\mathrm{InGaAs}$ & $2297-2392$ & parallel to slit \\
\hline 7 & $730-990$ & $\mathrm{Si}$ & $805-908$ & 45 degrees to long direction of slit \\
\hline
\end{tabular}

Table 3: Characteristics of the PMD detectors.

It is well known that the circular polarisation component appearing from atmospheric light can safely be taken as zero. Optical components are described to operate on these Stokes vectors as 4 by 4 Mueller matrices, for example for an aluminium mirror:

$$
\begin{gathered}
\mathrm{I}^{\prime} \\
\mathrm{Q}^{\prime} \\
\mathrm{U}^{\prime} \\
\mathrm{V}^{\prime}
\end{gathered}=\left(\begin{array}{cccc}
M_{11} & M_{12} & 0 & 0 \\
M_{12} & M_{11} & 0 & 0 \\
0 & 0 & M_{33} & M_{34} \\
0 & 0 & -M_{34} & M_{33}
\end{array}\right)\left(\begin{array}{l}
\mathrm{I} \\
\mathrm{Q} \\
\mathrm{U} \\
\mathrm{V}
\end{array}\right)
$$

In nadir mode this general structure is mantaned throughout all optical components and the intensity measured by the detectors is described by:

$$
'=M_{11} * I+M_{12} * Q
$$

In practice this means that by adding measurement information about the $Q$ Stokes parameter by measuring the polarisation component parallel to the entrance slit for a number of wavelength bands the instrument can be corrected for the polarisation dependence in the nadir mode. This is reflected in the PMD design (see also table 3) and in the calibration approach.

In the SCIAMACHY limb mode the convenient structure of these matrices is broken by the orientation of the ASM with respect to the ESM. In order to describe this in the Mueller matrix formalism a rotation matrix has to be included, resulting in the following result:

$M_{\text {elevation }} \otimes$ Rotation $(\alpha) \otimes M_{\text {azimuth }}=\left(\begin{array}{cccc}M_{11} & M_{12} & M_{13} & 0 \\ M_{21} & M_{22} & M_{23} & M_{24} \\ M_{31} & M_{32} & M_{33} & M_{34} \\ 0 & M_{42} & M_{43} & M_{44}\end{array}\right.$

The light intensity measured by the detectors (array detectors and polarisation detectors) is in principle now described by:

$$
I^{\prime}=M_{11} * I+M_{12} * Q+M_{13} * U
$$


depending on $I, Q$, and $U$. Whereas for the nadir mode it can be shown that only the Stokes parameters $I$ and $Q$ are important to radiometrically calibrate the instrument, when non-depolarising optics are assumed, information about the $U$ Stokes parameter is required in the limb mode to achieve an accurate radiometric calibration. For these reasons the $7^{\text {th }}$ PMD detector, which measures the $U$-Stokes-component of the incident light, was introduced into the instrument design for the limb mode. The limb radiometric calibration and the calibration algorithms are much more complicated than for nadir because of this, requiring more calibration information of the instrument.

The thermal-background radiation levels of the instrument itself in the infrared channels 7 and 8 have a significant impact on the performance of these channels in terms of signal levels and measurement noise. For this reason the optical bench is cooled down to $253 \mathrm{~K}$ using a passive cooler plate. At this temperature the thermal background radiation is drastically reduced, resulting in much better performance of the infrared channels 7 and 8 . All array detectors are cooled to their operational temperatures (see table 1) by means of a radiant cooler via a thermal bus that consists of an ammonia-filled heatpipe. At each detector the cooling capacity is tuned to the figures listed in table 1 . Due to the above thermal design features the instrument can obviously not be fully operated under ambient temperature and pressure conditions. Furthermore, from earlier similar satellite instrument programmes it is well-known that various optical components employed in the SCIAMACHY design change their transmission or reflective properties in a polarisation dependent way from air to vacuum conditions and with temperature. Outgassing of the optical components is also known to cause similar effects. The calibration and performance verification approach of SCIAMACHY has been such that all calibration and performance verification measurements, which are anticipated to change from air to vacuum and at different array detector and optical bench temperature conditions, are measured under flight representative thermal-vacuum conditions at nominal angles of the scan mirrors (e.g. in nadir and limb). These thermal-vacuum measurements have been performed in the socalled OPTEC thermal-vacuum facility with accompanying optical stimuli. In this facility the optical bench can be cooled down to its in-flight temperature of -17.9 degrees Celsius (this is also the temperature of the PMD detectors) and the array detectors can be cooled down to their operational temperatures (table 1). Since the final in-flight array detector temperatures are not yet known accurately, the relevant calibration parameters were calibrated at 2 detector temperature levels. Figure 5 shows this temperature dependence for the radiometric calibrations. The effect can be as large as $3 \%$ per Kelvin. Some calibration and performance parameters are expected to change from air-to-vacuum and at different temperatures as a result of optical component outgassing, prism dispersion changes, and changes in optical characteristics. However, since the OPTEC vacuum chamber is equipped with two optical windows for nadir and limb, only enabling illumination conditions for nominal scan mirror angles, the scan angle dependence of the radiometric calibration of the instrument can not be measured under these thermal-vacuum conditions. Instead, the polarisation-dependent reflective properties of the bare scan mirrors (i.e. without the rest of the spectrometer), which are not expected to change from air to vacuum or with temperature, are calibrated under ambient conditions. This approach has been accounted for in the radiometric calibration formalism.

The instrument is also equipped with two internal calibration lamps: a spectral line source (SLS) and a white light source (WLS). The wavelength and spectral lineshape calibrations of the instrument can be found from the SLS measurements and the results can be compared to the results obtained from solar Fraunhofer and atmospheric trace gas features in flight. The internal SLS is a PtCrNe hollow cathode discharge lamp. A typical SLS spectrum as recorded by the instrument is shown in figure 6, which shows that sufficient spectral emission lines are available in all channels, even though the line density decreases rapidly towards the infrared. In the infrared channels 7 and 8 a plasma discharge continuum can be observed as well, with emission lines superimposed on it. The response in the ultraviolet channels 1 and 2 has been zoomed in figure 6 . The WLS is a quartz-tungsten-halogen lamp which emits a Planck-like black-body emission spectrum with an equivalent temperature of about $3000 \mathrm{~K}$. The light bulb has been specifically manufactured for the SCIAMACHY project in order to transmit ultraviolet light down to $240 \mathrm{~nm}$. The instrument response of the internal WLS in the ultraviolet channel 1 is shown in figure 7 , whereas figure 8 shows the response for all channels. The purpose of the internal WLS is manifold. Firstly, it can be used to monitor optical or other efficiency changes quite accurately, even if neither the lamp nor its power supply has been specifically designed for this purpose. Secondly, array detector pixel-to-pixel gain variations can be calibrated by providing a smooth input spectrum. Thirdly, an effect known as variable etalonning can be calibrated and monitored using the WLS. The Si array detectors of channels 1-5 are coated with a 3 micrometer thick $\mathrm{SiO}_{2}$ protective layer, which causes spectral features in the transmission response by interference (fixed etalon). This can for example be observed in figures 7 and 8 . Very small thickness variations of this layer, in the order of a few tens of nanometer, caused by deposition of contaminants, most notably water-ice, can cause changes in the interference patterns resulting in efficiency changes of similar spectral signature as the fixed etalon and with transmission amplitudes up to several percents. This effect of variable etalonning can be observed and calibrated using the internal WLS, has been found to be small in SCIAMACHY during the on-ground testing phases under thermal-vacuum conditions, whereas its impact is much larger in the Global Ozone Monitoring Experiment (GOME), the scaled-down predecessor of SCIAMACHY, which flies since May 1995 on 
board of the ESA-ERS2 satellite. THis difference can be understood in terms of design differences of the focal plane assemblies. Finally, the WLS is used for calibrating and monitoring the NDF characteristics and the array detector temperature characteristics (see figure 5).

In this document a number of calibration and instrument performance characteristics of the SCIAMACHY instrument are discussed, both for the ambient scan mirror and the thermal-vacuum periods.

\section{CALIBRATION OF SCAN MIRRORS AND DIFFUSER}

The calibration of both scan mirrors, including the on-board diffuser was performed under ambient conditions at the calibration facility at TPD in Delft, The Netherlands, on unit level, i.e. the scan mirror modules were not integrated into the optical bench. This calibration includes the polarisation-dependent radiometric calibration and the calibration of the angular dependence of both mirrors. Three operational modes were calibrated:

1) Nadir, only ESM

2) Limb, ASM plus ESM

3) Diffuser, ASM plus on-board diffuser

The measurements are performed using either one or two polarisers to present polarised light to the mirrors or to measure polarised light coming from the mirrors (or both). Five different polarisation directions are necessary to calibrate the instrument: unpolarised $(u)$, s-polarised $(s)$, p-polarised $(p),+45$-polarised (4), and -45 -polarised $(m)$. For example, a measurement with the first polariser in the s-polarised position and the second polariser in the +45 -polarised direction is called $s 4$. The required calibration keyparameters are:

nadir us,up,pp,p4

limb us,up,ss,pp,4s,4p,ms,mp,44,s4,p4,sp,ps

diffuser $u s, u p$

As can be observed from the above list much less scan mirror calibration data is required for the nadir mode as compared to the limb mode. The reason for this lies in the orientation of both scan mirrors in the limb mode, as explained in the polarisation description of section 1. Some typical results of the scan mirror calibration are shown in figures 9 (nadir angular dependence as compared to theory [ASAP]), figure 10 (limb reflectance) and figure 11 (diffuser 'reflectance').

\section{THERMAL-VACUUM CALIBRATION AND PERFORMANCE}

The absolute radiance calibrations for nadir and limb (figure 12) and the absolute irradiance calibration for the sun-overdiffuser mode (figure 13) were performed under thermal-vacuum conditions. In figure 12 the absolute radiance calibration for the GOME instrument is depicted for comparison.

The wavelength calibration, instrument spectral response functions, and spectral resolution are derived from measurements with internal and external ( $\mathrm{PtCrNe}, \mathrm{HgAr}$, low pressure $\mathrm{Xe}$ ) spectral line sources. Fourth order polynomials are used to describe the dispersion behaviour of each channel. The spectral response functions for all channels are best described by hyperbolic functions of the type

$$
y=\frac{a^{2}}{b^{2}+(x-c)^{2}}+\frac{d^{2}}{b^{2}+(x-c)^{4}}
$$

The polarisation calibration consists of a part measured under thermal-vacuum conditions with the scan mirrors in their nominal nadir and limb positions and the ambient scan mirror and diffuser calibration described above. Two polarisation parameters are of direct interest for the calculation of the calibration keyparameters: $\eta$ and $\xi$, defined as follows:

$$
\begin{aligned}
& \eta=\frac{\text { array detector response to } \mathrm{s}-\text { polarised light }}{\text { array detector response to } \mathrm{p}-\text { polarised light }} \\
& \xi=\frac{\text { polarisation detector response to } \mathrm{p}-\text { pol. light }}{\text { array detector response to } \mathrm{p}-\mathrm{pol} \text { light }}
\end{aligned}
$$

The $\eta$ parameter is measured with an external white light source and a Brewster polariser and the $\xi$ parameter with a monochromator and a Brewster polariser, because the PMD detectors are broadband detectors and the $\xi$ parameter is 
required at a fine wavelength grid. Both experimental setups are part of the dedicated and fully commissioned optical stimuli setup that pertains to the OPTEC setup. Both $\eta$ and $\xi$ parameters need to be corrected for setup artefacts. The $\eta$ and $\xi$ parameters can be combined with the angular-dependent scan mirror and diffuser calibration key parameters to yield a full set of all required polarisation calibration keyparameters. The results for the $\eta$ and $\xi$ calibration parameters are shown in figures 14 and 15 , respectively. The spectral sensitivity curves of the polarisation detectors, shown in figures 16 and 17 (uncorrected for monochromator output), can cross over the spectral boundaries of the main optical channels, causing singularities in the $\xi$ parameter. For this reason multiple $\xi$ calibration parameters, made up of combinations with polarisation detector signals with various array detector channels over which the polarisation detector spectral responsivity curve extends, are calculated. The dotted line in figure 16 is the PMD \#7 curve, which corresponds in the spectral domain close to the PMD \#4 curve. The jump at $1650 \mathrm{~nm}$ is caused by a grating change of the monochromator and is not due to the instrument.

Furthermore, a number of so-called Brewster scan measurements have been performed. In this kind of measurement the polarisation direction is stepped from e.g. -90 degrees to +180 degrees in steps of 5 degrees and at each step the instrument response is measured. Figure 18 shows an example of such a measurement. In the figure the response of PMD's 6 and 7 are shown. It can be observed that the $6^{\text {th }}$ PMD measures p-polarised light (at about 1.5 radians), whereas the $7^{\text {th }}$ PMD measured +45 -polarised light (at about 2.3 radians), in agreement with the instrument design.

The spectral straylight is also calibrated and characterised under thermal-vacuum conditions. Because the spectral straylight can in principle be polarisation-dependent (originating from imperfect grating performance and light reflections), measurements were defined with different input polarisations to verify this, but almost no polarisation dependence of the spectral straylight was found in practice. Spectral straylight can be divided into spectral ghost straylight (localised on a few pixels) and uniform straylight (distributed over all pixels in a channel). No spectral ghosts were detected stronger than typically $0.05 \%$.

The Instantaneous Field Of View (IFOV) in cross-dispersion direction of all detectors (all pixels and PMD detectors) was also measured under thermal-vacuum conditions for the large and the small apertures. The IFOV size is an important calibration parameter since it defines the observed area on the earth or in the atmosphere. From the optical design the large IFOV size used for nadir and limb observations is $1.8 \times 0.045$ degrees $^{2}$ and $0.72 \times 0.045$ degrees $^{2}$ for the direct sun observations using the small aperture. The measurement results are shown in figures 19,20 and 21,22 for the large and small apertures, respectively. The measured responses are shown as a function of illumination angle within the IFOV in crossdispersion direction for a number of pixels in various array detector channels. From these figures the $50 \%$ response points can be determined, indicated by the vertical bars in the figures, and the IFOV size and alignment in cross-dispersion direction can be measured for all pixels in the array detectors and for all PMD detectors. The latter results are shown in figures 20 and 22 (neglect the cases in figures 20 and 22 where the results are off-scale in the figures, this is due to measurement artefacts and not a result of instrument performance, because optimised optical setups and light sources have been employed for ultraviolet and infrared light). It can be seen from figures 20 and 22 that measured results are very close to these design requirements. Furthermore, it can be observed that all detectors are almost perfectly aligned with respect to each other within 0.006 degrees. The results for the small aperture show a classical diffraction pattern, which changes considerably with wavelength (figure 21). Provided that the direct sun measurement conditions are always similar, this diffraction pattern cancels from the measurement ratio's. Otherwise, the measurement results can be deconvoluted using the response curves like shown in figure 21 . The IFOV size in dispersion direction has been verified under ambient conditions using a theodolite.

\section{CONCLUSIONS}

The SCIAMACHY instrument has been calibrated and characterised on ground both under ambient and thermal-vacuum pressure and temperature conditions and a number of results are described in this document. The instrument design has been outlined briefly and important radiometric and spectral calibration and performance parameters have been discussed. The ambient scan mirror and diffuser results and the polarisation calibration parameters are shown. Finally, the instantaneous field of view characterisation results have been discussed. The as-measured instrument calibration and performance results compare well to the design values.

\section{ACKNOWLEDGEMENT}

The author is grateful for the support of the SCIAMACHY project team. 

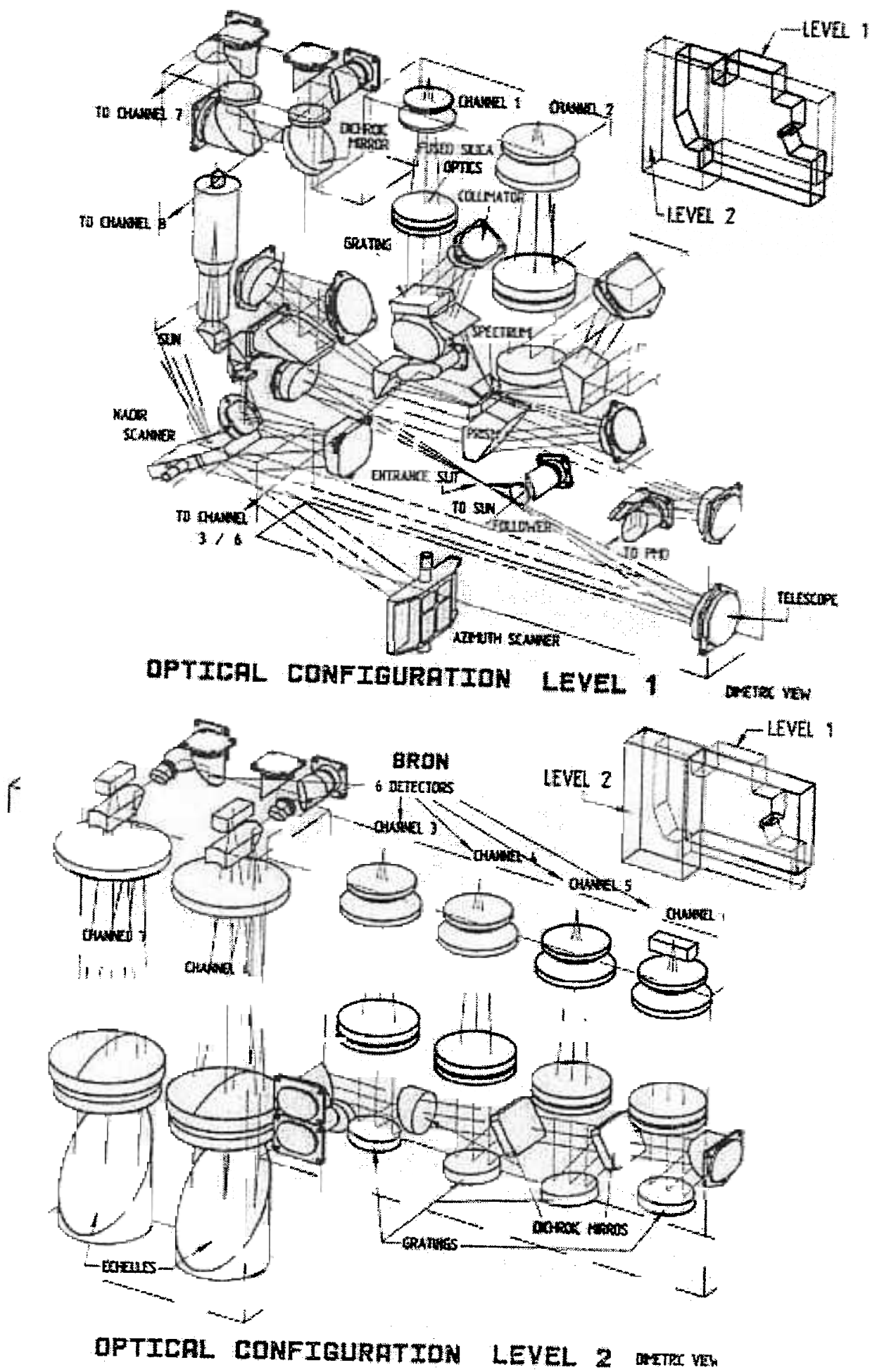

Figure SCIAMACHY optical layout. 
SCIAMACHY Sun-over-Diffuser Mode

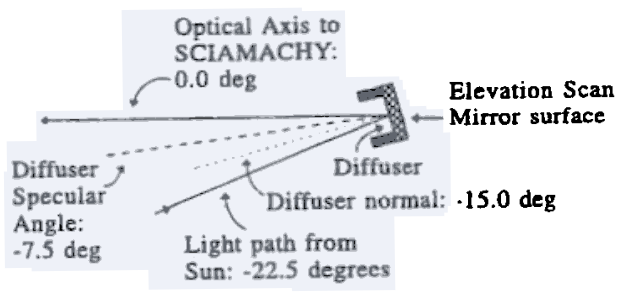

Figure 2: Diffuser geometry for sun-over-diffuser measurements.

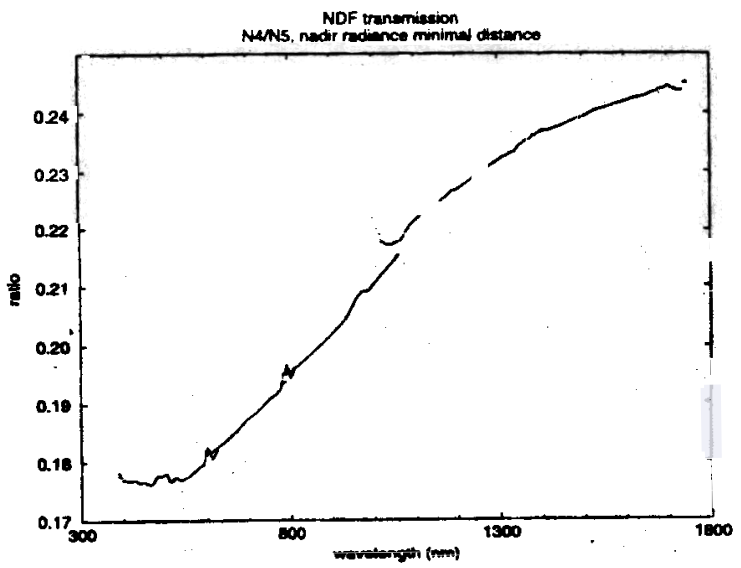

Figure 3: Neutral density filter transmission.

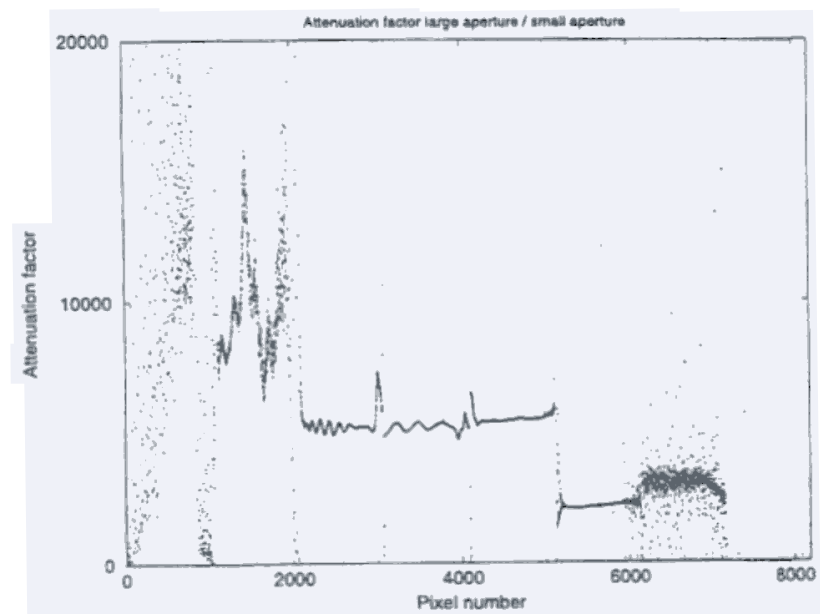

Figure 4: Ratio of large aperture over small aperture showing signal attenuation of the small aperture.

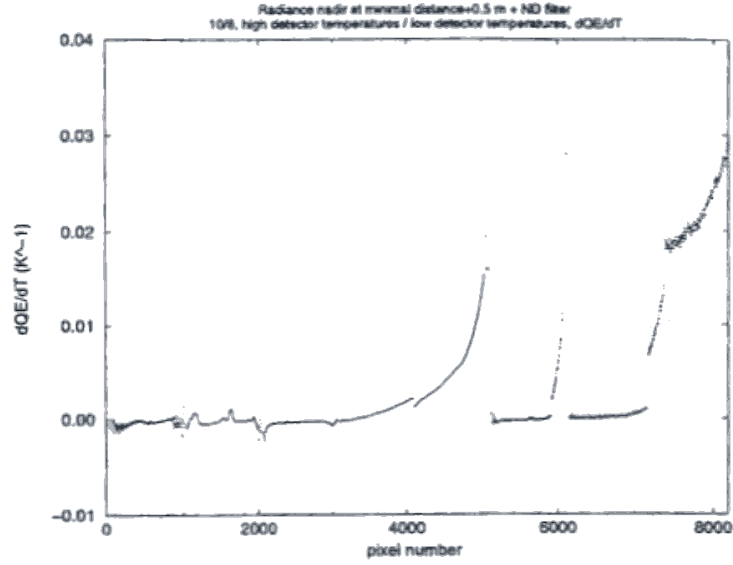

Figure 5: Array detector radiometric sensitivity dependence on detector temperature.

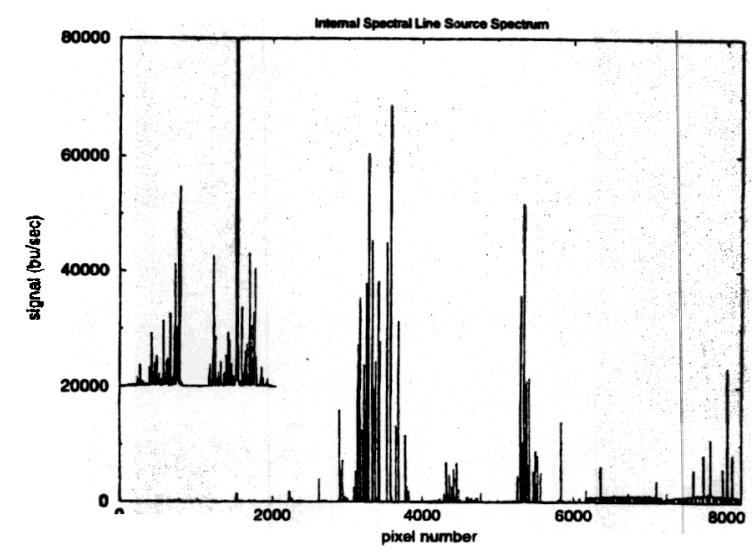

Figure 6: Internal spectral line source measurement.

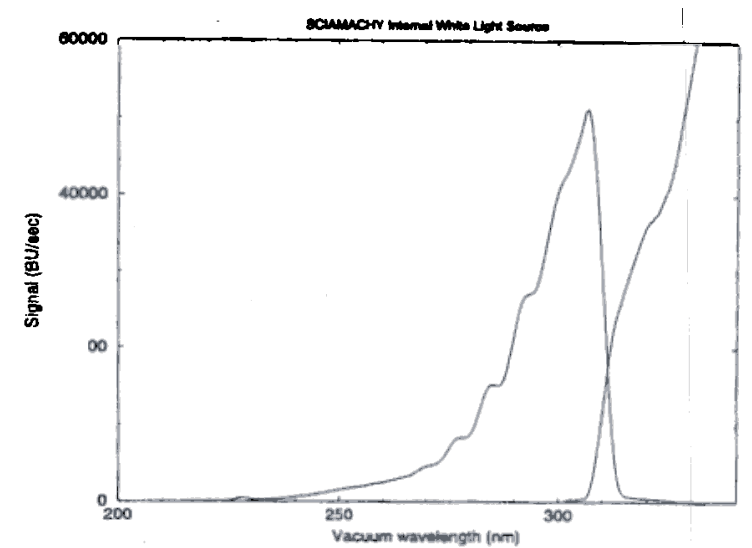

Figure 7: Internal white light source measurement in the ultraviolet in channel 1. 


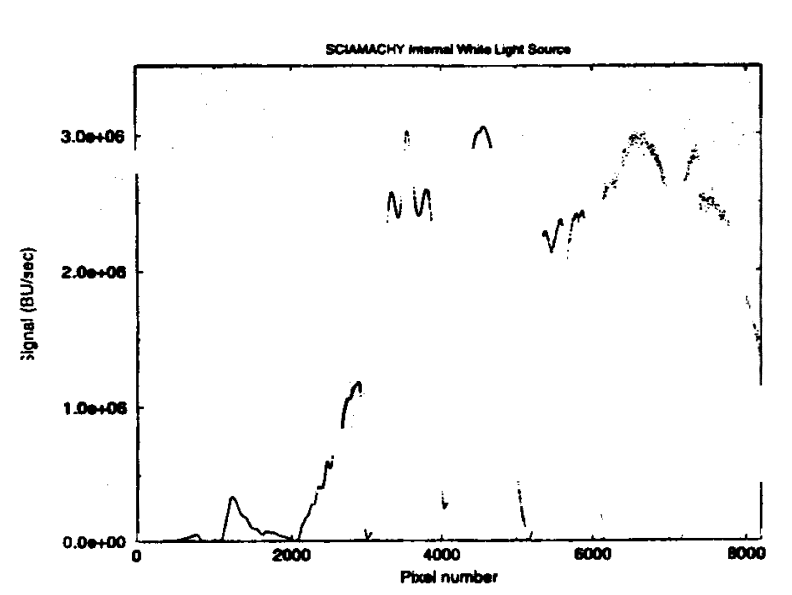

Figure 8: Internal white light source measurement for all channels.

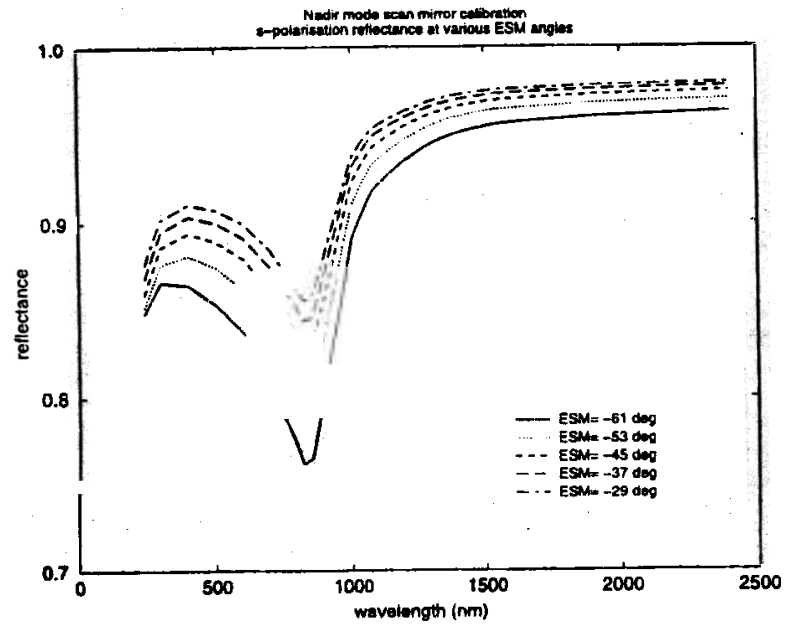

Figure 9: Ambient scan mirror and diffuser calibration: nadir mode scan mirror reflectance example.

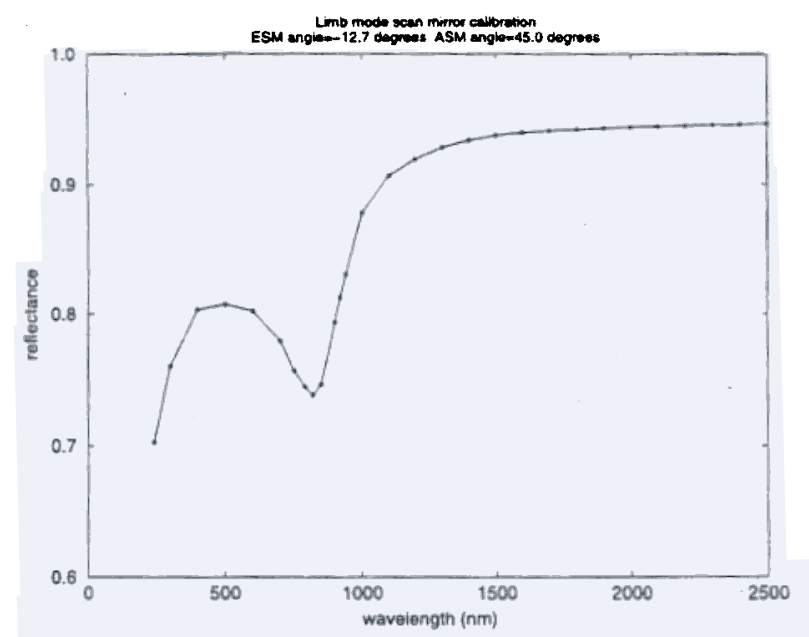

Figure 10: Ambient scan mirror and diffuser calibration: limb mode reflectance example.

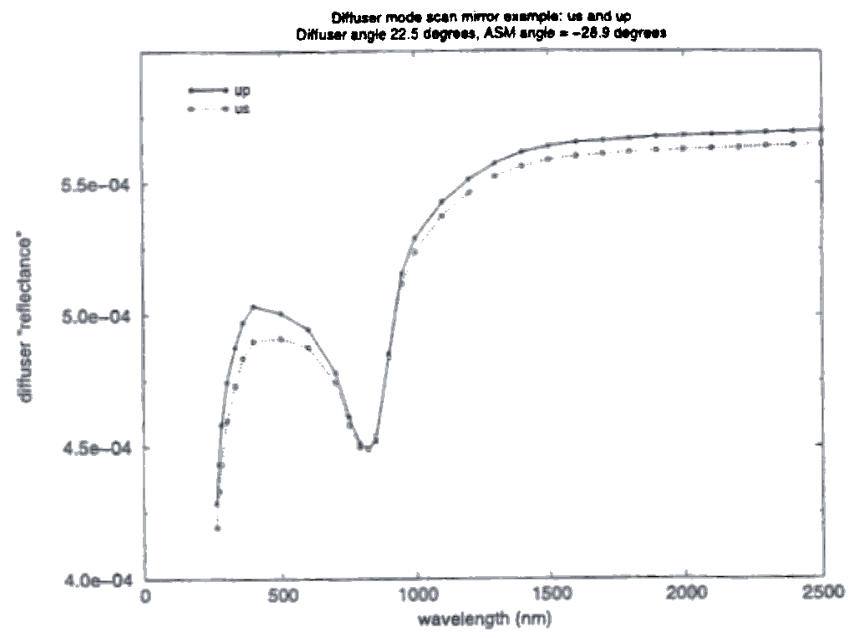

Figure 11: Ambient scan mirror and diffuser calibration: diffuser mode measurement example.

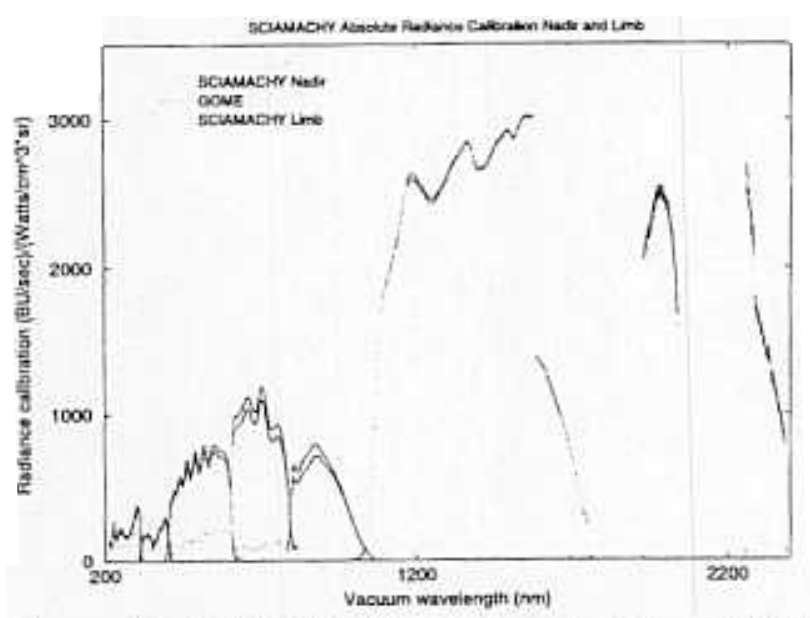

Figure 12: SCIAMACHY nadir and limb absolute radiance calibration. The dotted line is the GOME-1 absolute radiance calibration.

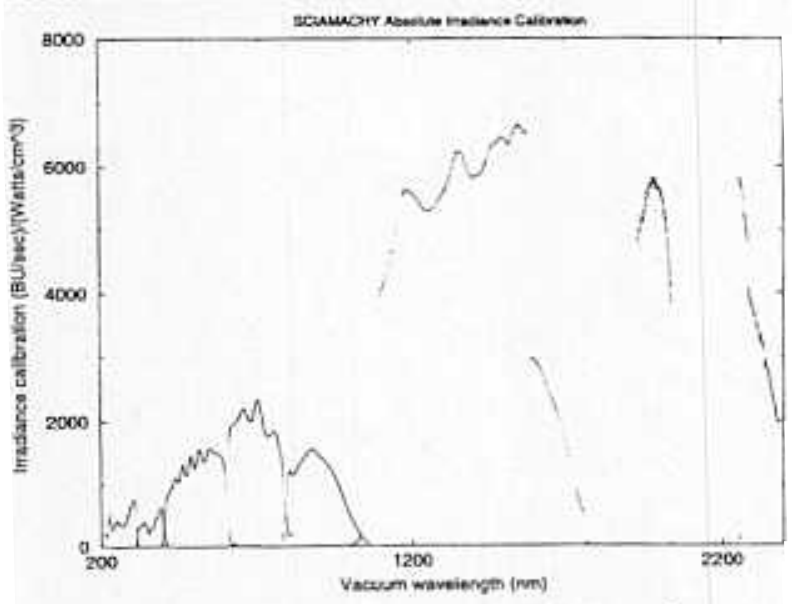

Figure 13: Absolute irradiance calibration results. 


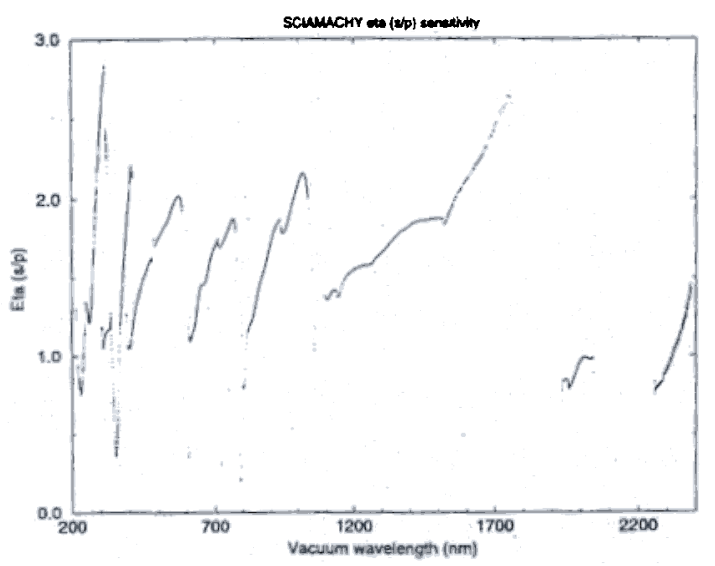

Figure 14: $\eta$ calibration keyparameter.

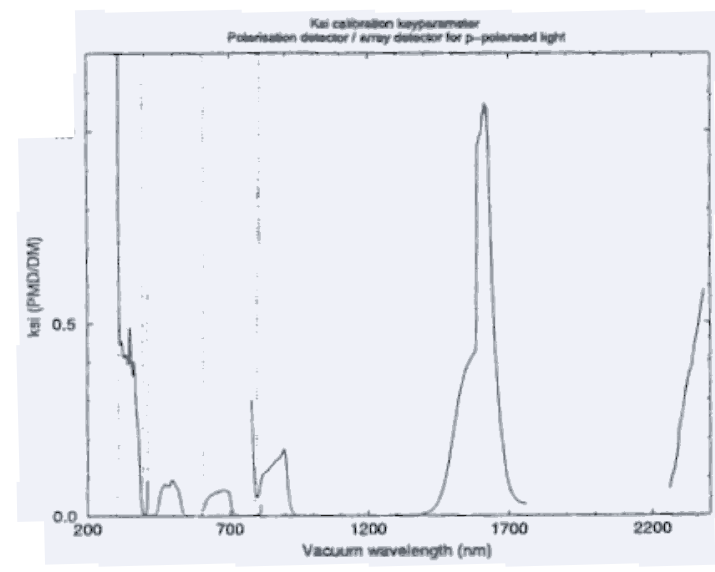

Figure $15: \xi$ calibration keyparameter
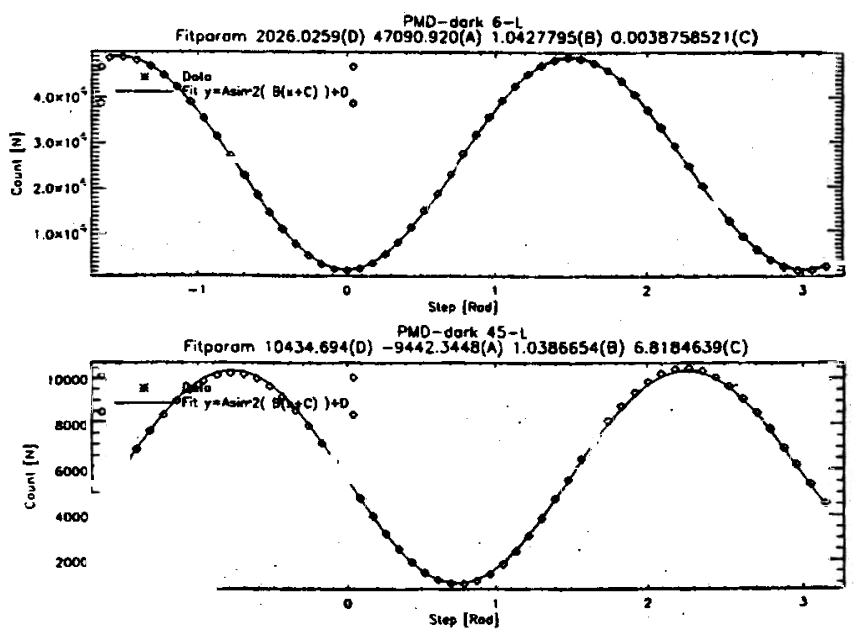

Figure 18: Brewster scan measurement example for polarisation detectors 6 and 7 .

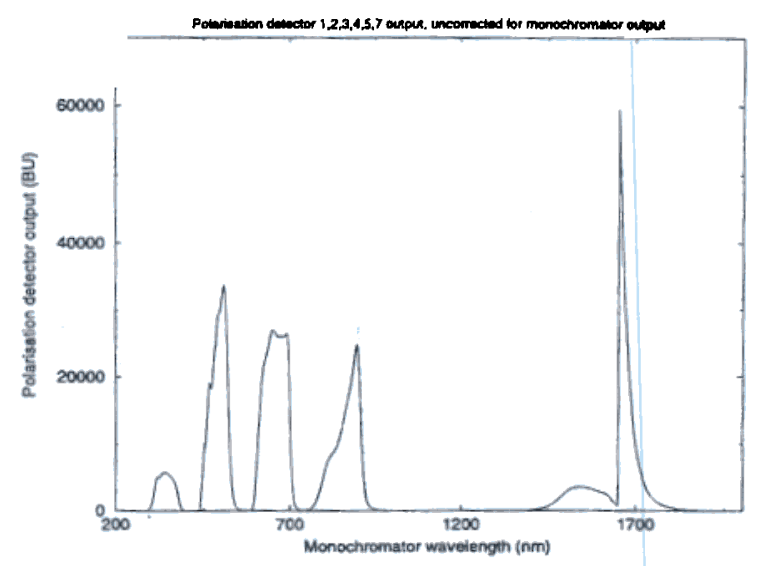

Figure 16: Spectral response of the polarisation detectors $1-5$ and 7.

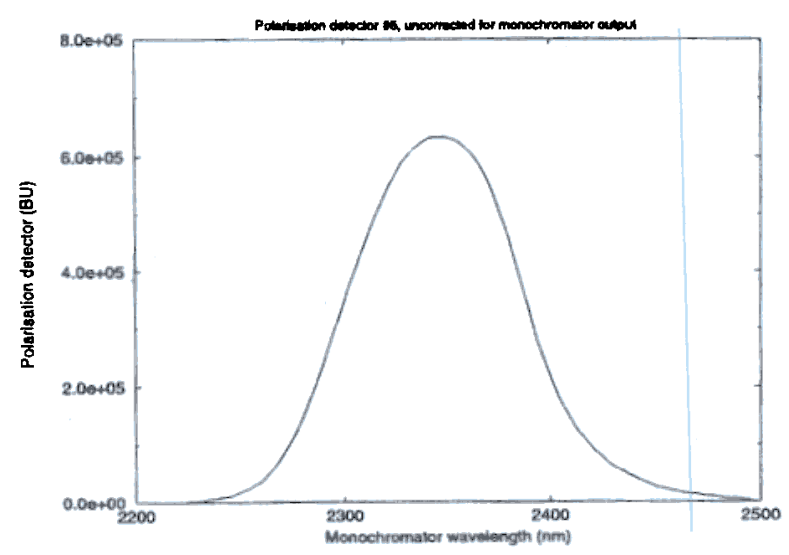

Figure 17: Spectral response of polarisation detector 6. 

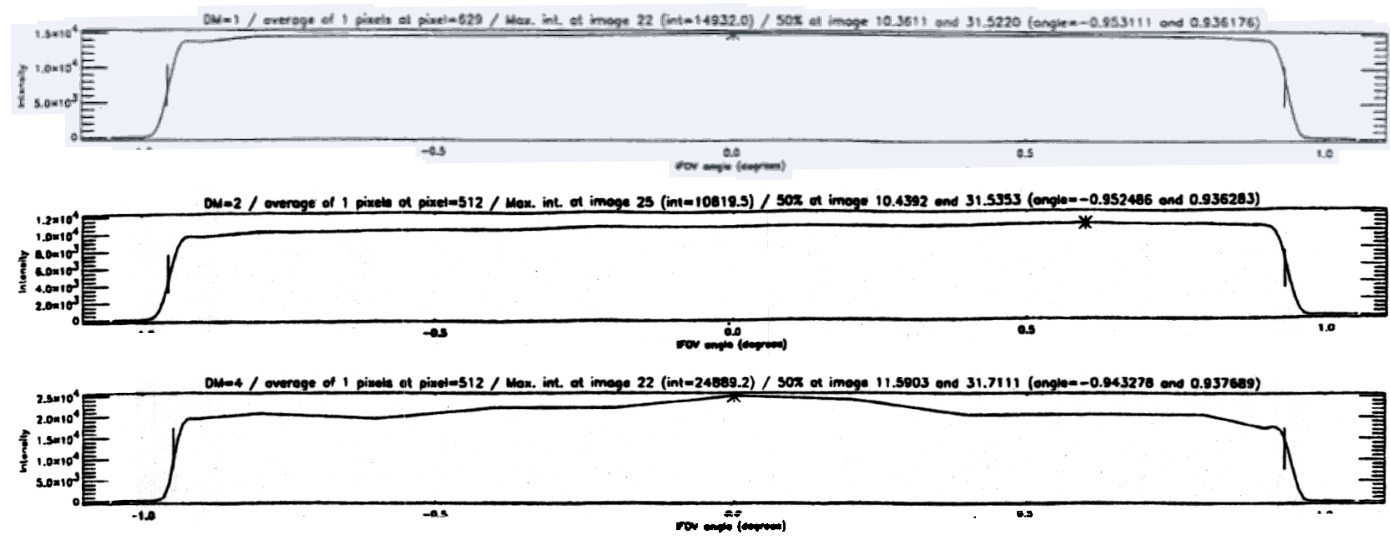

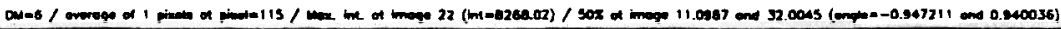

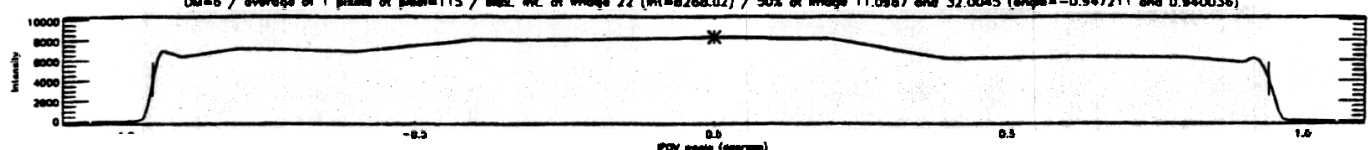

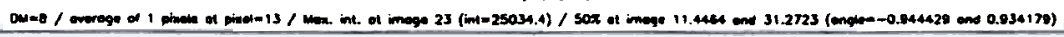

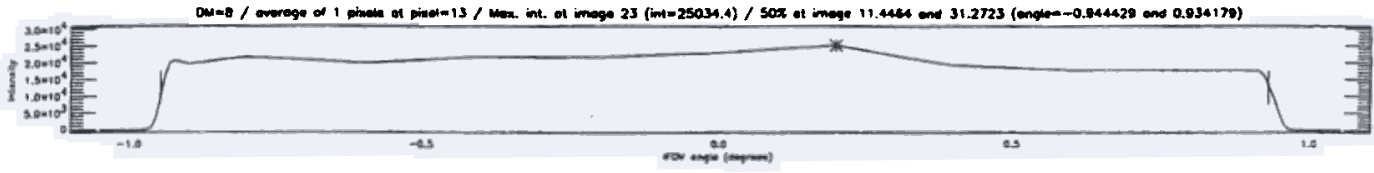

Figure 19: IFOV in cross-dispersion direction for the large aperture for a number of array detector channels
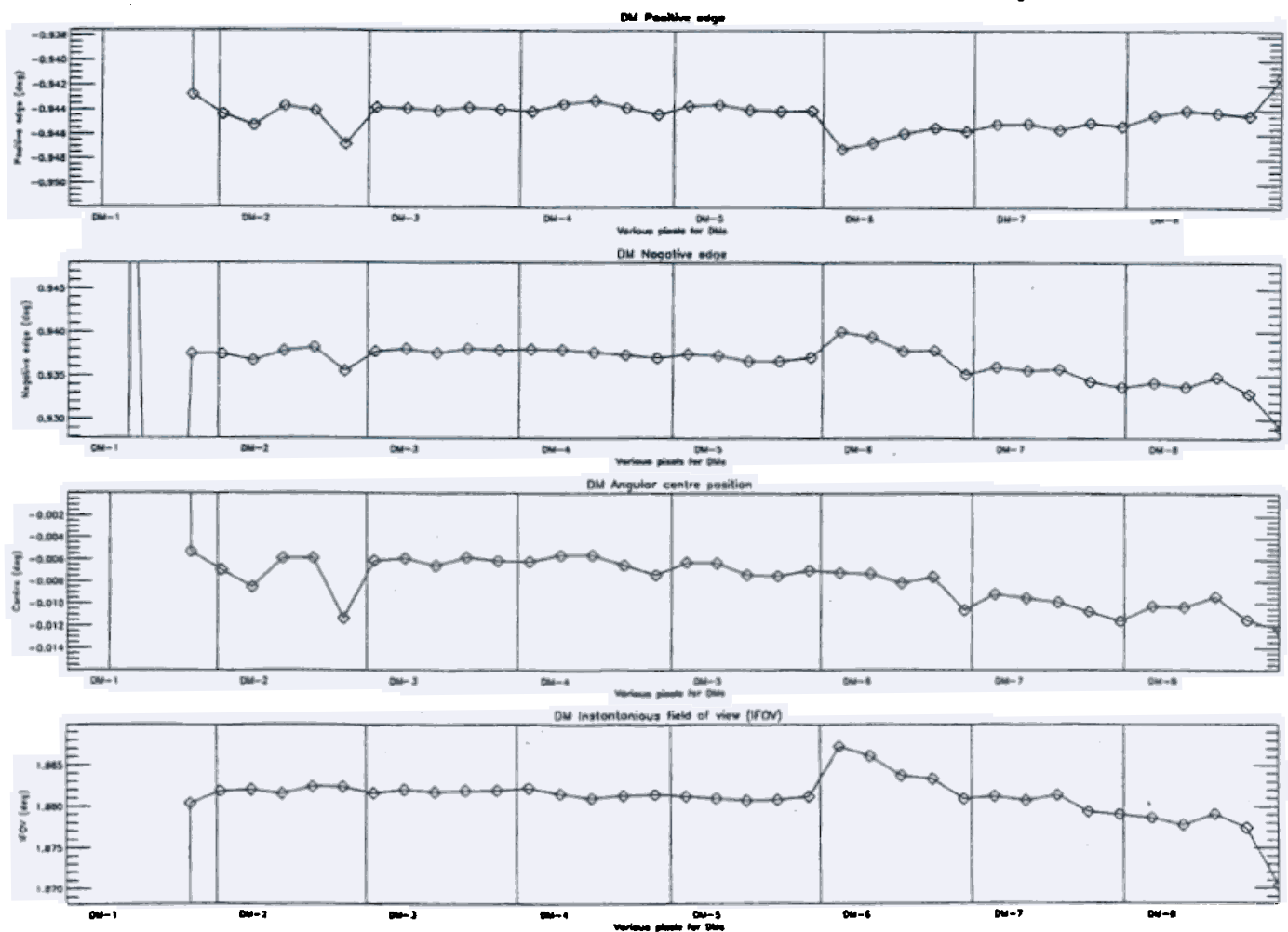

Figure 20: IFOV in cross-dispersion direction for the large aperture for a number of array detector channels: IFOV size and alignment. 

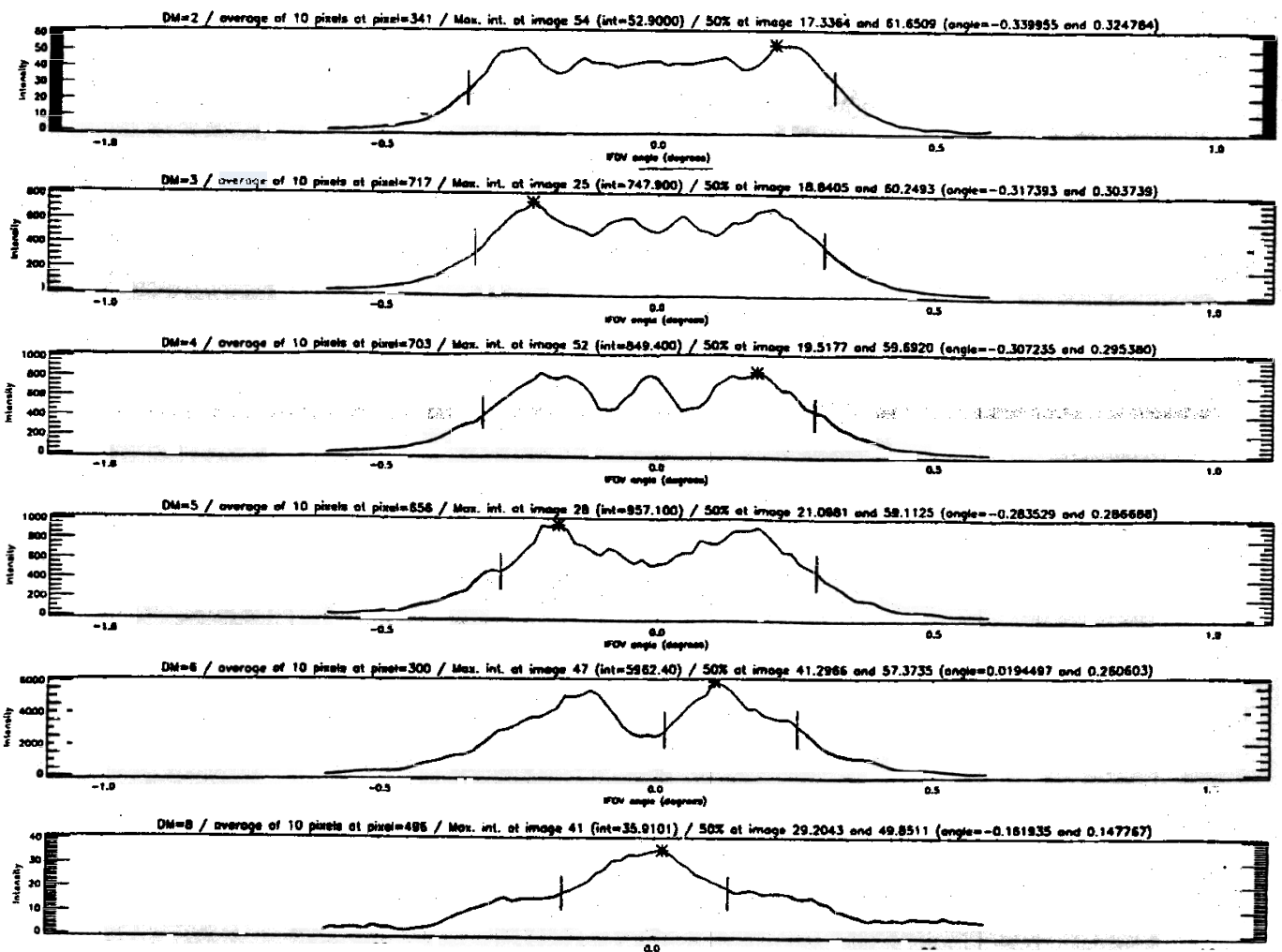

Figure 21: IFOV in cross-dispersion direction for the small aperture for a number of array detector channels
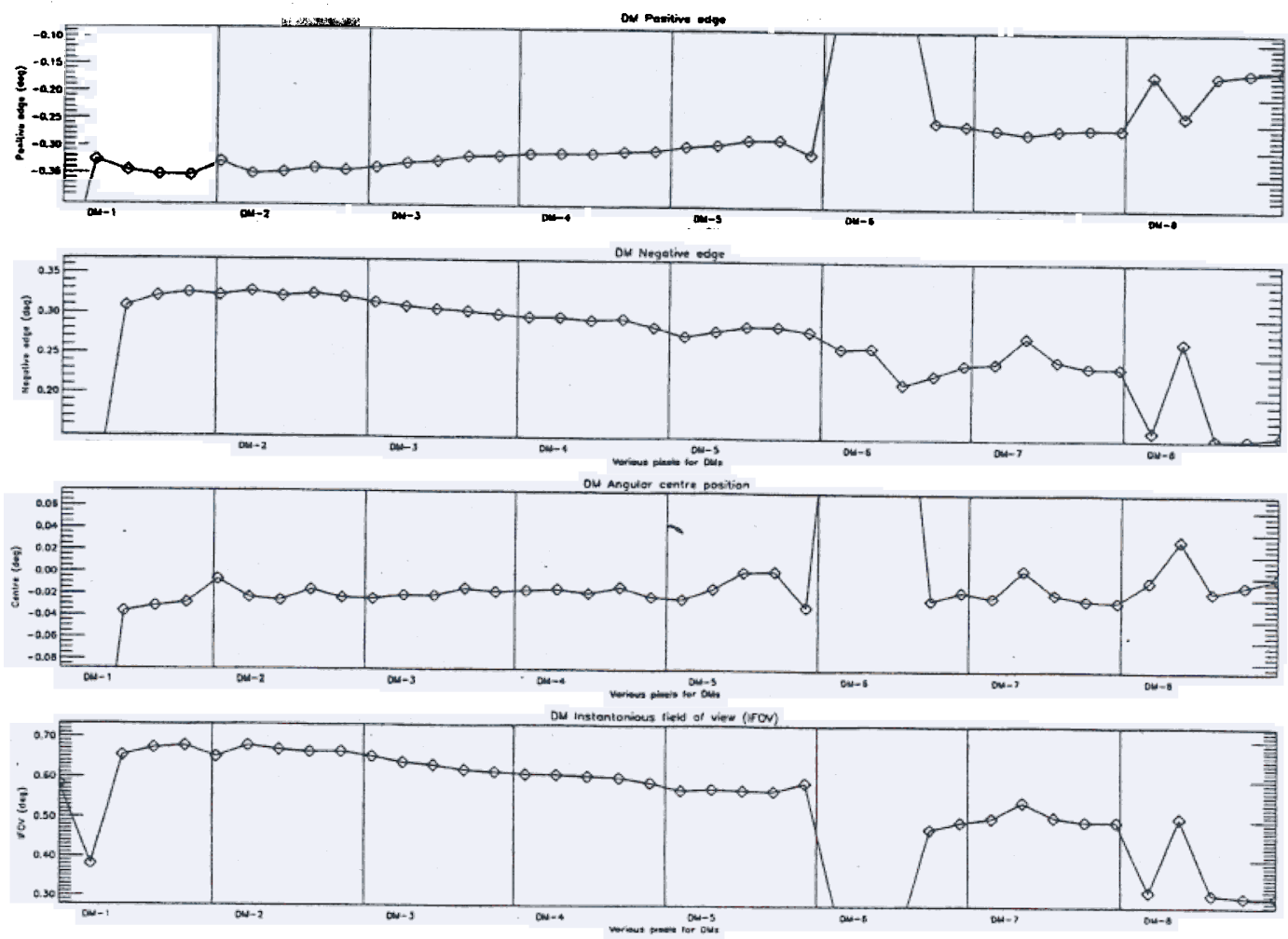

Figure 22: IFOV in cross-dispersion direction for the small aperture for a number of array detector channels: IFOV size and alignment. 